\title{
A Simple Analytical Expression for the Gradient Induced Potential on Active Implants During MRI
}

\author{
Esra A. Turk*, Emre Kopanoglu, Sevin Guney, K. Emre Bugdayci, Y. Ziya Ider, \\ Vakur B. Erturk, and Ergin Atalar
}

\begin{abstract}
During magnetic resonance imaging, there is an interaction between the time-varying magnetic fields and the active implantable medical devices (AIMD). In this study, in order to express the nature of this interaction, simplified analytical expressions for the electric fields induced by time-varying magnetic fields are derived inside a homogeneous cylindrical volume. With these analytical expressions, the gradient induced potential on the electrodes of the AIMD can be approximately calculated if the position of the lead inside the body is known. By utilizing the fact that gradient coils produce linear magnetic field in a volume of interest, the simplified closed form electric field expressions are defined. Using these simplified expressions, the induced potential on an implant electrode has been computed approximately for various lead positions on a cylindrical phantom and verified by comparing with the measured potentials for these sample conditions. In addition, the validity of the method was tested with isolated frog leg stimulation experiments. As a result, these simplified expressions may help in assessing the gradient-induced stimulation risk to the patients with implants.
\end{abstract}

Index Terms-Active implantable medical devices (AIMD), gradient fields, magnetic resonance imaging (MRI), stimulation risk.

\section{INTRODUCTION}

A LTHOUGH magnetic resonance imaging (MRI) is known to be a very safe diagnostic technique, patients with active implantable medical devices (AIMD) are generally not allowed to be scanned because of the undesirable interaction between the electromagnetic field generated by the MRI scanner and AIMD. While the effects of static magnetic and radiofrequency electromagnetic fields have been widely studied, the interaction

Manuscript received March 8, 2012; revised June 4, 2012; accepted July 12, 2012. Date of publication August 7, 2012; date of current version September 14,2012 . Asterisk indicates corresponding author.

${ }^{*} \mathrm{E}$. A. Turk is with the Department of Electrical and Electronics Engineering and the National Magnetic Resonance Research Center, Bilkent University, Ankara 06800, Turkey (e-mail: esra@ee.bilkent.edu.tr).

E. Kopanoglu and E. Atalar are with the Department of Electrical and Electronics Engineering and the National Magnetic Resonance Research Center, Bilkent University, Ankara 06800, Turkey (e-mail: emrek@ee.bilkent.edu.tr; ergin@ee.bilkent.edu.tr).

S. Guney is with the Faculty of Medicine, Gazi University, Ankara 06800, Turkey (e-mail: sguney@ gazi.edu.tr).

K. E. Bugdayci is with the Department of Molecular Biology and Genetics, Bilkent University, Ankara 06800, Turkey (e-mail: bugdayci@fen. bilkent.edu.tr).

Y. Z. Ider and V. B. Erturk are with the Department of Electrical and Electronics Engineering, Bilkent University, Ankara 06800, Turkey (e-mail: ider@ee.bilkent.edu.tr; vakur@ee.bilkent.edu.tr).

Digital Object Identifier 10.1109/TBME.2012.2212190 between the implants and the gradient magnetic field has not been studied in depth.

In MRI, gradient waveforms are usually designed as pulses. Their ramp up and down times are usually considered as dead times and minimized for maximum performance. On the other hand, a rapidly switched gradient magnetic field induces an electric field inside the body that may cause nerve stimulation [1]. In the presence of an AIMD, the risk of stimulation increases [2]. In particular, when a cardiac pacemaker or an implantable cardioverter-defibrilator is present in the patient during an MRI examination, possibility of cardiac arrest is a very serious concern [3], [4].

To investigate the peripheral nerve stimulation risk and the threshold value, in the absence of any metallic implant, theoretical and experimental studies have been carried out [5]-[12]. In these studies, electric field distributions are analyzed for theoretical explanations of the stimulation risk. For the electric field measurements, field probe is used in [12]. However, it is useful only for the measurement of the induced electric field at the body boundary. Furthermore, to define the induced electric field, both computational methods such as finite difference time domain [8] and analytical calculations have been performed using inhomogeneous and homogeneous human models [5], [13], [14]. The studies [2]-[4], [15] show that a time-varying magnetic field causes stimulation by inducing an electric field on an AIMD inside the human body. However, in [15] and [2], no experimental verification is performed and in [4], experiments are only performed for the Helmholtz coil. On the other hand, in [3], experiments were performed on six mongrel dogs and the induced current was measured with a current recorder. However, no analytical explanation about the stimulation risk is carried out.

In a safety analysis of AIMD during an MRI examination, a generic and simple formulation of the induced potential on electrodes of AIMD has a critical importance. This will give an insight into the worst case conditions for implants. With this simple formulation of the induced potential on electrodes and the knowledge of the lead impedance, the appropriate filter for leads to protect the patient from the stimulation risk during MRI may also be designed. Although in [13], the induced electric and magnetic field expressions were derived for a homogeneous cylindrical body model so that the induced potential on an electrode can be found, the provided expressions involve complicated Fourier integrals to be calculated numerically. Hence, they are not suitable for obtaining a generic and simple induced potential expression.

Therefore, in this study, we provide closed-form expressions of electric and magnetic fields for a linear gradient field formed 
by an infinitely long cylindrical gradient coil. With the simplified field expressions, induced potential causing stimulation is estimated by assuming a unipolar pacing model. We conducted phantom experiments to compare the difference between our estimated and actually measured potential values, and we also tested the accuracy of our expressions in estimating the stimulation risk with ex vivo frog nerve experiments. Experimental results show that, using the simplified expressions, we can determine the voltage induced on the implant lead if the path of the implant lead is known.

\section{THEORY}

To estimate the stimulation risk, we need to calculate the induced voltage on the implant lead, which can be deduced from the induced electric field distribution. During the ramping up and ramping down periods of the gradient fields, an induced electric field $\vec{E}$ is set up in the medium. If there is an implant lead in the medium, insulated except for the tips and extended in the direction of $\vec{E}$, then charge accumulates at the tips immediately to generate an opposing electric field. The total electric field is equal to the sum of the magnetically induced electric field and the charge induced electric field. Near the tip of the lead the charge induced electric field is the dominating one and furthermore because it has steep variation near the tip it is the cause of stimulation of a nearby nerve membrane [16].

The amount of current leaving the lead and flowing in the medium due to the charge-induced electric field is determined by the charge induced potential difference between the two ends of the implant lead divided by the impedance which is the sum of the contact impedances and the equivalent impedance of the medium. This voltage difference can be calculated by integrating the charge-induced electric field along the path of the lead. This integral on the other hand is equal to the negative of the integral of the magnetically induced electric field along the path of the lead, since inside the lead the total electric field is almost zero. This stimulation can be likened to the working of a unipolar pacing system, where there is a lead connecting the only electrode within the heart with a metal casing supplying power.

In [13], to calculate the electric field $\vec{E}$, first the scalar potential $V$, and the vector potential $\vec{A}$ inside the gradient coil have been solved by applying an appropriate boundary condition on the surface of a cylindrical volume. It is assumed that the conductivity of the volume is uniform and nearly equal to the average of the conductivity of tissues inside the body as done in similar studies on the subject. Moreover, since the gradient magnetic field is a low-frequency field, skin depth is assumed to be much larger than the physical size of the stimulated tissue region, which means that the induced current inside the body is not a source to generate magnetic field. Similarly, for low frequencies, the displacement current is also ignored. Under these assumptions the scalar and the vector potential equations have been simplified and utilized to provide the electric and the magnetic field expressions inside the body in [13]. However, the field expressions provided in [13] are defined in terms of their
Fourier transforms and the coil current distribution is required for the computational analysis of the field distributions.

In this study, we provide generic simplified electric and magnetic field expressions that do not require the current distribution to be known in advance. In addition to the same assumptions with [13], we also assumed that the gradient coil is infinitely long, in other words, gradient field is linear in everywhere.

According to the general principle of the target field method, for a specified target field on a cylindrical surface with radius $c$ (i.e., $\rho=c$ ), the Fourier transform of the current flowing in $\phi$-direction can be found as the following [17]:

$$
J_{\phi}^{(m)}(k)=-\frac{B_{z}^{(m)}(c, k)}{\mu_{0} k a I_{m}(k c) K_{m}^{\prime}(k a)} \quad c \leq a
$$

where $B_{z}^{(m)}(c, k)$ is the Fourier transform of the target magnetic field in $z$-direction over a cylindrical surface with radius $c$; $I_{m}(k c)$ and $K_{m}(k a)$ are the first and the second kind modified Bessel functions of order $m ; k$ is spatial frequency; $a$ is the radius of the coil; and $\mu_{0}$ is the permeability of the medium.

As the design parameter, the $z$-component of the magnetic field is given as $B_{z}=x G_{x}+y G_{y}+z G_{z}$, where $G_{x}, G_{y}$, and $G_{z}$ are the gradient fields in the $x$-, $y$-, and $z$-directions, respectively. For imaging purposes, $G_{x}, G_{y}$, and $G_{z}$ are constant within the volume of interest. In order to define the target magnetic fields in the $z$-direction for $x$-, $y$-, and $z$-gradient coils, the $g(z)$ function that describes the field variation in the $z$-direction can be added to the predefined $B_{z}$ field expression [18]. Simplification of the field expressions is performed by using these target fields.

For $x$-gradient coil, the target field is taken as $B_{z}(c, \phi, z)=$ $G_{x} x g(z)=G_{x} c \cos \phi g(z)$. To find $J_{\phi}^{(m)}(k)$, first the Fourier transform, $B_{z}^{(m)}(c, k)$ for the given target field is defined as follows:

$$
\begin{aligned}
B_{z}^{(m)}(c, k) & =\frac{1}{2 \pi} \int_{-\infty}^{\infty} \int_{-\pi}^{\pi} e^{-i m \phi} e^{-i k z} B_{z}(c, \phi, z) d \phi d z \\
& =\frac{1}{2 \pi} \int_{-\infty}^{\infty} \int_{-\pi}^{\pi} e^{-i m \phi} e^{-i k z} G_{x} g(z) c \cos \phi d \phi d z \\
& =G_{x} c \frac{\delta_{-1 m}+\delta_{1 m}}{2} \int_{-\infty}^{\infty} g(z) e^{-i k z} d z \\
& =G_{x} c \frac{\delta_{-1 m}+\delta_{1 m}}{2} g(k)
\end{aligned}
$$

where $g(k)=\int_{-\infty}^{\infty} g(z) e^{-i k z} d z$, the Kronecker symbol $\delta_{j m}$ has the value 1 if $j=m$ and 0 otherwise, and $i=\sqrt{-1}$. By inserting this $B_{z}^{(m)}(c, k)$ field into (1), the expression for $J_{\phi}^{(m)}(k)$ is as follows:

$$
J_{\phi}^{(m)}(k)=-\frac{G_{x} c\left(\delta_{-1 m}+\delta_{1 m}\right) g(k)}{2 \mu_{0} k a I_{m}(k c) K_{m}^{\prime}(k a)} .
$$

Equation (3) is used in the field expressions defined for a cylindrical volume with radius $\rho_{0}$ given in [13], and the field components in the form of Fourier transforms are derived. Before starting the simplification, the inverse Fourier transform of each 
component is expressed as follows:

$$
\begin{aligned}
& E_{\rho}(\rho, \phi, z)=-\omega G_{x} \frac{1}{2 \pi} \sum_{m=-\infty}^{\infty} e^{i m \phi} \\
& \times \int_{-\infty}^{\infty} e^{i k z}\left(\delta_{-1 m}+\delta_{1 m}\right) \frac{m g(k)}{2 k^{2}} \frac{c}{I_{m}(k c)} \\
& \times\left(\frac{I_{m}(k \rho)}{\rho}-\frac{I_{m}\left(k \rho_{0}\right) I_{m}^{\prime}(k \rho)}{\rho_{0} I_{m}^{\prime}\left(k \rho_{0}\right)}\right) d k \\
& E_{\phi}(\rho, \phi, z)=-i \omega G_{x} \frac{1}{2 \pi} \sum_{m=-\infty}^{\infty} e^{i m \phi} \\
& \times \int_{-\infty}^{\infty} e^{i k z}\left(\delta_{-1 m}+\delta_{1 m}\right) \frac{g(k)}{2 k} \frac{c}{I_{m}(k c)} \\
& \times\left(I_{m}^{\prime}(k \rho)-\frac{m^{2} I_{m}\left(k \rho_{0}\right) I_{m}(k \rho)}{k^{2} \rho \rho_{0} I_{m}^{\prime}\left(k \rho_{0}\right)}\right) d k \\
& E_{z}(\rho, \phi, z)=-i \omega G_{x} \frac{1}{2 \pi} \sum_{m=-\infty}^{\infty} e^{i m \phi} \\
& \times \int_{-\infty}^{\infty} e^{i k z}\left(\delta_{-1 m}+\delta_{1 m}\right) \frac{m I_{m}\left(k \rho_{0}\right)}{2 k^{2} \rho_{0} I_{m}^{\prime}\left(k \rho_{0}\right)} \\
& \times \frac{c I_{m}(k \rho)}{I_{m}(k c)} g(k) d k \\
& B_{\rho}(\rho, \phi, z)=-i G_{x} \frac{1}{2 \pi} \sum_{m=-\infty}^{\infty} e^{i m \phi} \\
& \times \int_{-\infty}^{\infty} e^{i k z}\left(\delta_{-1 m}+\delta_{1 m}\right) \frac{c I_{m}^{\prime}(k \rho)}{2 I_{m}(k c)} \\
& \times g(k) d k, \\
& B_{\phi}(\rho, \phi, z)=G_{x} \frac{1}{2 \pi} \sum_{m=-\infty}^{\infty} e^{i m \phi} \\
& \times \int_{-\infty}^{\infty} e^{i k z}\left(\delta_{-1 m}+\delta_{1 m}\right) \frac{m c}{2 k \rho} \\
& \times \frac{I_{m}(k \rho)}{I_{m}(k c)} g(k) d k \text {. }
\end{aligned}
$$

Note that the expressions will be different than 0 , only for $m=1$ and $m=-1$ indices due to the Kronecker delta functions $\delta_{-1 m}$ and $\delta_{1 m}$.

The function $g(z)$, describing the $z$-variation of the magnetic field, has to be chosen as given in [17] to satisfy the current continuity condition. Accordingly, the function $g(z)$ used in this study is chosen as $g(z)=2 \sin c(2 z / b)-\sin c(z / b)$ and $|z|<b$ region of the function is shown in Fig. 1. The value of $b$ is related with the dimension of the coil in the $z$-direction.

Fourier transform of the gradient field $B_{z}^{(m)}(c, k)$ is proportional with the function $g(k)$ as given in (2). The Fourier transform of the $g(z)$ function used in this study is shown in Fig. 2. When left and right limits are equal to each other, $\lim _{b \rightarrow \infty} \int_{-\infty}^{\infty} g(k) f(k) d k=\lim _{k \rightarrow 0} f(k)$. Accordingly,

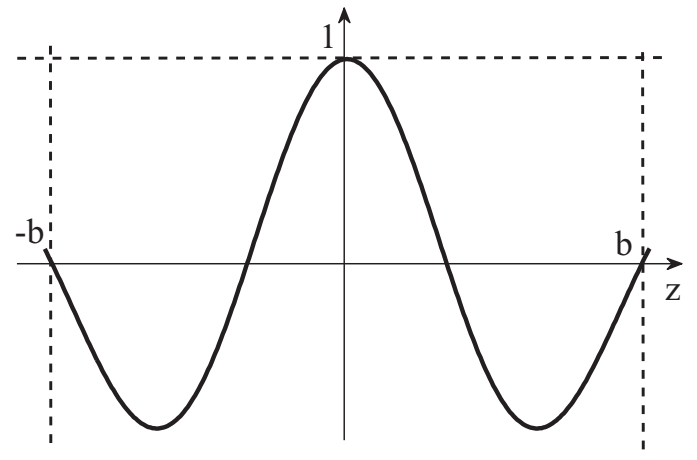

Fig. 1. Specified $g(z)$ field.

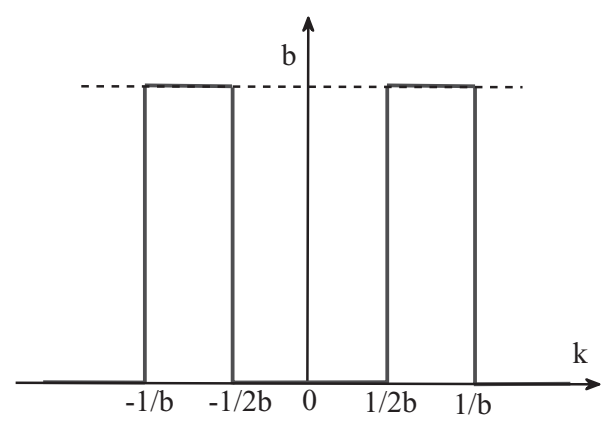

Fig. 2. Fourier transform of the specified $g(z)$ field. As $b \rightarrow \infty$, two pulses approach impulses at $0_{+}$and $0_{-}$. However, $g(k)$ remains equal to zero at $k=0$.

the field equations can be simplified as follows:

$$
\begin{aligned}
& E_{\rho}(\rho, \phi, z)=-i \omega c G_{x} \sin \phi\left[\lim _{k \rightarrow 0} e^{i k z} \frac{1}{k^{2} I_{1}(k c)}\right. \\
&\left.\times\left(\frac{I_{1}(k \rho)}{\rho}-\frac{I_{1}\left(k \rho_{0}\right) I_{1}^{\prime}(k \rho)}{\rho_{0} I_{1}^{\prime}\left(k \rho_{0}\right)}\right)\right] \\
&=-i \omega G_{x} \sin \phi\left(\frac{\rho_{0}^{2}-\rho^{2}}{4}\right) \\
& E_{\phi}(\rho, \phi, z)=-i \omega c G_{x} \cos \phi\left[\lim _{k \rightarrow 0} e^{i k z} \frac{1}{k I_{1}(k c)}\right. \\
& \times\left(I_{1}^{\prime}(k \rho)-\frac{I_{1}\left(k \rho_{0}\right) I_{1}(k \rho)}{k^{2} \rho_{0} \rho I_{1}^{\prime}\left(k \rho_{0}\right)}\right] \\
&=-i \omega G_{x} \cos \phi\left(\frac{\rho_{0}^{2}+\rho^{2}}{4}\right) \\
& E_{z}(\rho, \phi, z)=-\omega c G_{x} \sin \phi\left[\lim _{k \rightarrow 0} e^{i k z} \frac{I_{1}\left(k \rho_{0}\right) I_{1}(k \rho)}{k^{2} \rho_{0} I_{1}^{\prime}\left(k \rho_{0}\right) I_{1}(k c)}\right] \\
& B_{\phi}(\rho, \phi, z)=-i \omega G_{x} \sin \phi \rho z \\
& B_{\rho}(\rho, \phi, z)=-i G_{x} c \cos (\phi)\left[\lim _{k \rightarrow 0} e^{i k z} \frac{I_{1}^{\prime}(k \rho)}{I_{1}(k c)}\right] \\
&= G_{x} z \cos \phi \\
&\left.\lim _{k \rightarrow 0} e^{i k z} \frac{I_{1}(k \rho)}{k \rho I_{1}(k c)}\right] \\
&(11) \sin \phi .
\end{aligned}
$$


In the derivation of (9)-(13), derivatives of the modified Bessel functions are substituted with the appropriate recurrence relations for the modified Bessel functions [19]. Additionally, in order to simplify the limit operation, small argument approximation for the Bessel functions is used. Note that since the electric field components vanish with the first-order small argument approximation, the second order approximation is used for simplifications. For $y$ - and $z$-gradient coils, target fields are defined as $B_{z}(c, \phi, z)=G_{y} c \sin \phi g(z)$ and $B_{z}(c, \phi, z)=G_{z} z g(z)$, respectively. The field expressions for these gradient coils are also simplified in the same way applied to the $x$-gradient coil. The resultant electric and magnetic field expressions in the Cartesian coordinates, in time domain are obtained as follows [20]:

$$
\begin{aligned}
& E_{x}=\frac{x y}{2} \frac{d G_{x}}{d t}+\frac{\rho_{0}^{2}-x^{2}+y^{2}}{4} \frac{d G_{y}}{d t}+\frac{y z}{2} \frac{d G_{z}}{d t} \\
& E_{y}=\frac{-\rho_{0}^{2}-x^{2}+y^{2}}{4} \frac{d G_{x}}{d t}-\frac{x y}{2} \frac{d G_{y}}{d t}-\frac{x z}{2} \frac{d G_{z}}{d t} \\
& E_{z}=-y z \frac{d G_{x}}{d t}+x z \frac{d G_{y}}{d t} \\
& B_{x}=z G_{x}-\frac{x}{2} G_{z} \\
& B_{y}=z G_{y}-\frac{y}{2} G_{z} \\
& B_{z}=x G_{x}+y G_{y}+z G_{z} .
\end{aligned}
$$

Note that (14)-(19) are obtained for a homogeneous cylindrical volume with the assumption that the gradient coil is infinitely long.

With these simplified electric field expressions, the induced voltage on the implant lead can be calculated approximately by integrating tangential $E$ field over the length of the lead.

\section{EXPERIMENTAL RESULTS}

\section{A. Experimental Setup}

In this study, both phantom and ex vivo experiments are performed with a Siemens Magnetom TimTrio $3 \mathrm{~T}$ system. In both experiments, a fixed MRI sequence is applied and the changes are observed. In the MRI sequence, no RF pulses are used. The magnitude of the gradient pulses is set to $30 \mathrm{mT} \cdot \mathrm{m}^{-1}$ with a ramp up and down time of $170 \mu \mathrm{s}$. The pulse duration is set to $5 \mathrm{~ms}$. In the sequence, there is a 5 -ms gap between each gradient pulse. In phantom experiments, the implant is aligned along the $z$-axis and the $x$-axis in order to verify the accuracy of the equations for the field variations in the $x$ - and $z$-directions. Forty different implant lead positions along the $z$-axis are considered for the $e x$ vivo experiments. $x-, y$-, and $z$-positions of the leads are determined using the MR magnitude images. Approximate induced potential on the lead is computed theoretically by integrating tangential $E$ field over the lead according to the position data.

Fig. 3 illustrates the cylindrical plexiglass phantom with a diameter of $30 \mathrm{~cm}$ and a length of $50 \mathrm{~cm}$ used in the experiments. Wires acting as an implant lead are fixed at different positions in the phantom as shown in Fig. 3. The diameter of the wire is $0.8 \mathrm{~mm}$. The wires are insulated without shielding. One tip of the wire is left uninsulated and the other tip going out from the

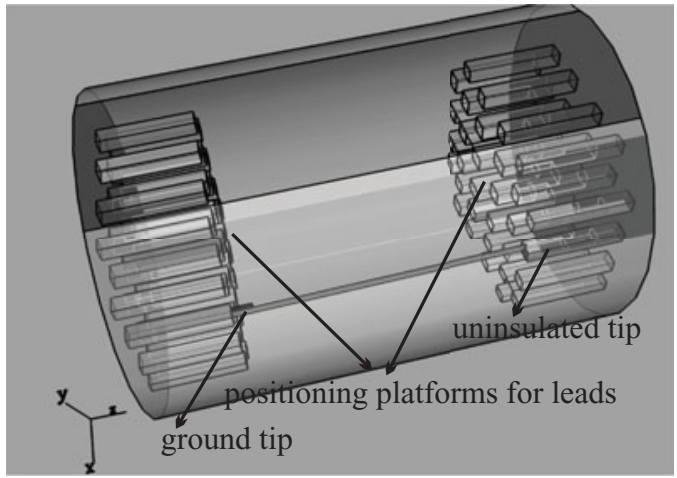

Fig. 3. Cylindrical plexiglass phantom with a diameter of $30 \mathrm{~cm}$ and a length of $50 \mathrm{~cm}$ and the positioning of the lead.

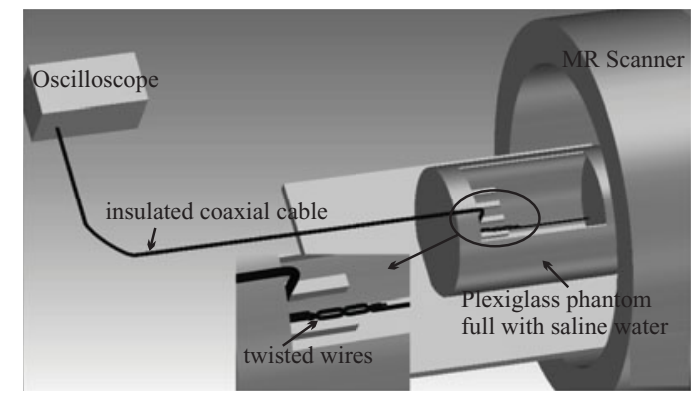

Fig. 4. Experimental setup: position of the wires, phantom and the oscilloscope.

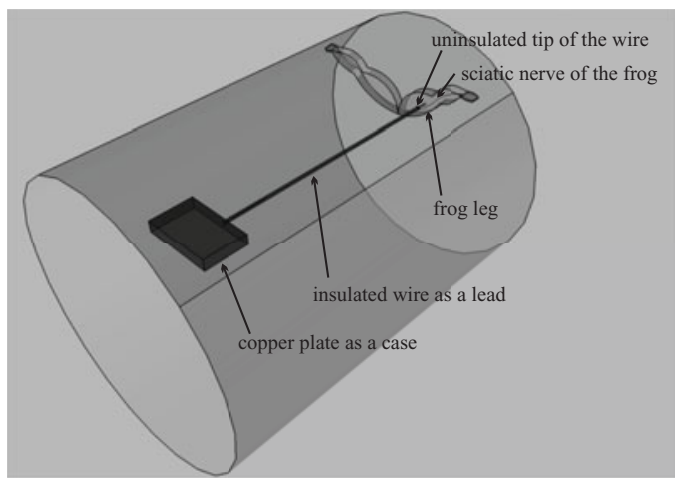

Fig. 5. Location of the frog leg and the lead inside the phantom.

phantom is connected to an oscilloscope probe. There is another wire attached to this wire that acts as a ground which is taken as a reference level. As an oscilloscope, Agilent InfiniiVision DSO7032A is used.

The voltage is carried by insulated coaxial cable to the oscilloscope. Insulated wire outside the phantom is twisted in order to ensure that the measurement is only coming from the lead inside the phantom. The experimental setup is shown in Fig. 4. For different implant lead positions, MR images of the phantom are taken and the signal waveform observed from the oscilloscope is stored. For each lead position, the peak voltage values observed in the oscilloscope are compared with theoretically computed voltage values for the respective lead positions.

A model of the ex vivo experiment setup is shown in Fig. 5. These experiments are performed using the sciatic nerves of 


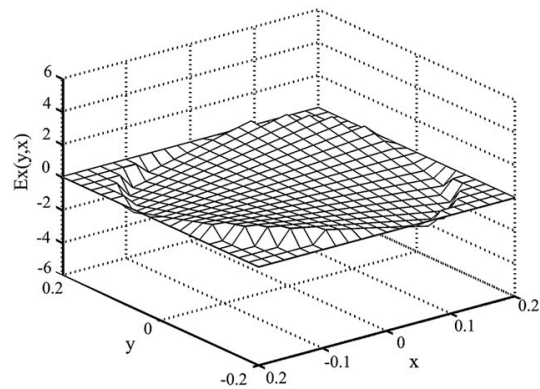

(a)

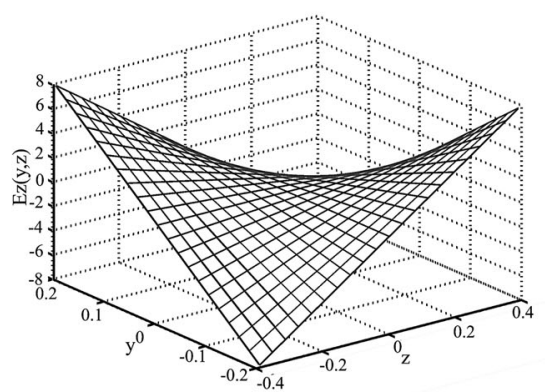

(c)

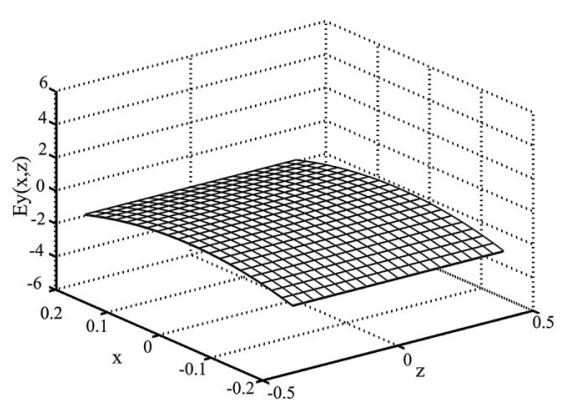

(b)

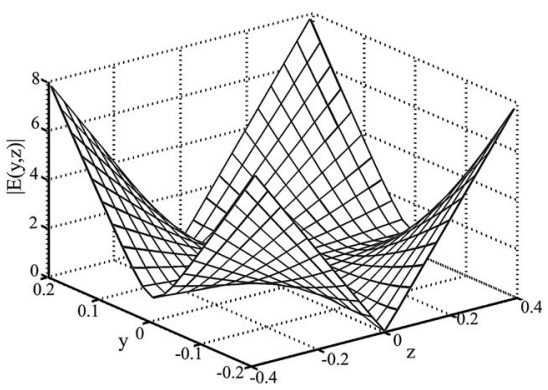

(d)

Fig. 6. $E$-field distribution formed by the equation of our study for $x$-gradient coil. (a) $E_{x}(y, x)$ for $z=0$. (b) $E_{y}(x, z)$ for $y=0$. (c) $E_{z}(y, z)$ for $x=0$. (d) $|E(y, z)|$ for $x=0$.

frogs. The nerve is kept alive inside Ringer's solution. One tip of a wire is soldered to a piece of copper plate, this plate emulates the pulse generator when there is no electrical component between the case and the lead, in other words when there is a short circuit between the case and the lead. The other tip of the wire touches the sciatic nerve of the frog as shown in Fig. 5. Only the tip touching the nerve is left uninsulated (emulates the electrode). The same cylinder used in phantom experiments is filled with Ringer's solution instead of saline water, and the frog leg and the wire are fixed inside the phantom.

To determine the threshold voltage value that stimulates the frog nerve, the same signal waveform observed during the phantom experiments is generated with two signal generators outside the MR scanner. The voltage is applied to the nerve with the same insulated wire used in the experiments. By changing only the amplitude of the signal, the minimum voltage value that stimulates the nerve (i.e., the minimum voltage value at which a muscular contraction is observed visually) is determined and defined as the threshold voltage value. In the experiments under MR scanner for different implant lead positions, the stimulation of the frog nerve is observed visually. For each implant lead position, with the help of the MR images, the induced voltage values are computed and compared with the threshold voltage.

\section{B. Results and Discussion}

By using MATLAB (Mathworks, Natick, MA), the electric field distributions are obtained by solving the simplified field expressions for an $x$-gradient coil with a diameter of $0.65 \mathrm{~m}$, $20 \mathrm{mT} \cdot \mathrm{m}^{-1}$ gradient magnitude and $100 \mathrm{~T} \cdot \mathrm{m}^{-1} \cdot \mathrm{s}^{-1}$ gradient switching rate, which are the same as the parameters used in [13].
Fig. 6 shows these electric field distributions for a conducting cylinder with 0.195 -m radius.

In the linear region of the gradient field, the obtained field patterns show similar characteristics with those given in [13]. In [13], the peak value of $|E|$ for the $x$-coil with a gradient switching rate of $100 \mathrm{~T} \cdot \mathrm{m}^{-1} \cdot \mathrm{s}^{-1}$ is calculated as $5.25 \mathrm{~V} / \mathrm{m}$, whereas at the same location, this value is found to be $6.3 \mathrm{~V} / \mathrm{m}$ using the simplified expressions. In [5], this peak value is calculated as $4.2 \mathrm{~V} / \mathrm{m}$. For the $z$-coil, the peak value of $|E|$ is calculated as $4 \mathrm{~V} / \mathrm{m}$ in our study and in [13], it is $3.53 \mathrm{~V} / \mathrm{m}$.

In the phantom experiments, the measured voltage value for each lead position is compared with the analytically computed voltage values. Fig. 7(a) shows the comparison of the calculated and the measured voltage values when the $x$-gradient coil is active and the implant leads are aligned in the $z$-axis along the body. Fig. 7(b) shows the same comparison for the activation of $y$-gradient coil. Unity line is shown to indicate the difference between the expected and the measured values.

According to these results, when the lead is aligned along the $z$-direction, the root-mean-square error between the calculated and the measured voltages is calculated as $26 \mathrm{mV}$. The error may be due to the fact that in the course of deriving the analytical expressions, both the gradient coils and the phantom are assumed to be infinitely long. However, the lengths are obviously finite in the experiments. For the $z$-gradient coil, the simplified field expressions show that $E_{z}$ is expected to be zero. In the measurements, for the $z$-gradient coil, the voltage level is in $5-10 \mathrm{mV}$ range and noisy, so it is classified as an error.

Fig. 8 shows the comparison of the calculated and the measured voltage values when $z-, y$-, and $x$-gradient coils are active, respectively, and the implant leads are aligned in the $x$-axis along the body. According to these results when the lead is along the 


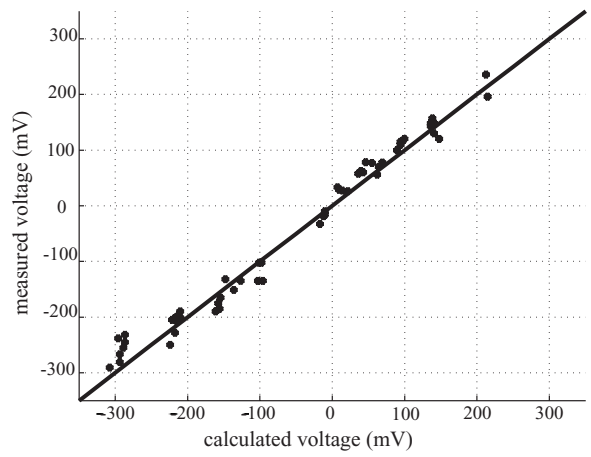

(a)

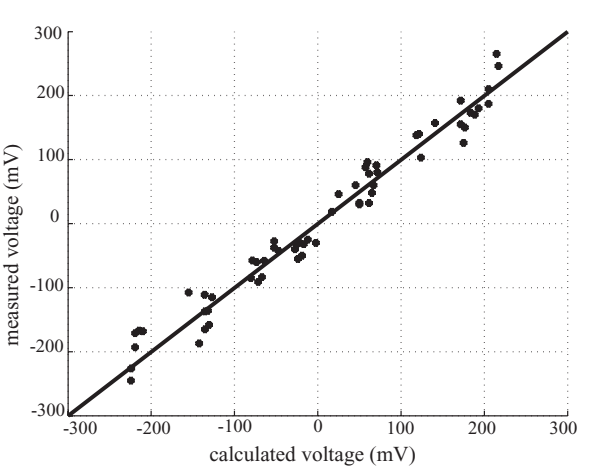

(b)

Fig. 7. Comparison of the calculated and the measured voltage values for the activation of: (a) $x$-gradient coil and (b) $y$-gradient coil. (Note that implant leads are aligned in the $z$-axis along the body)

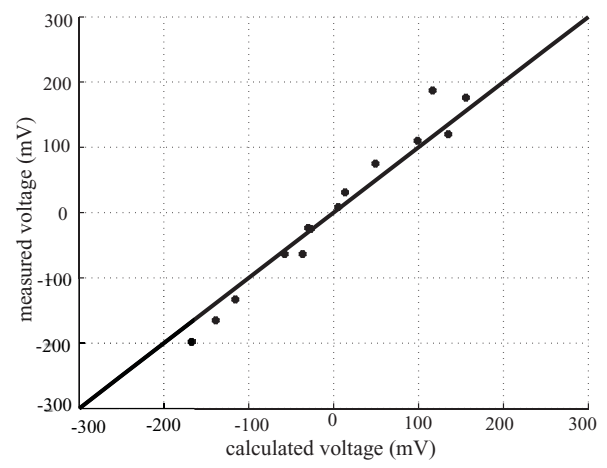

(a)

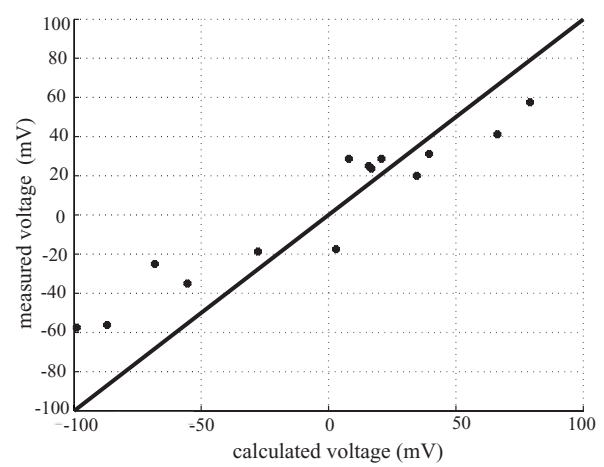

(b)

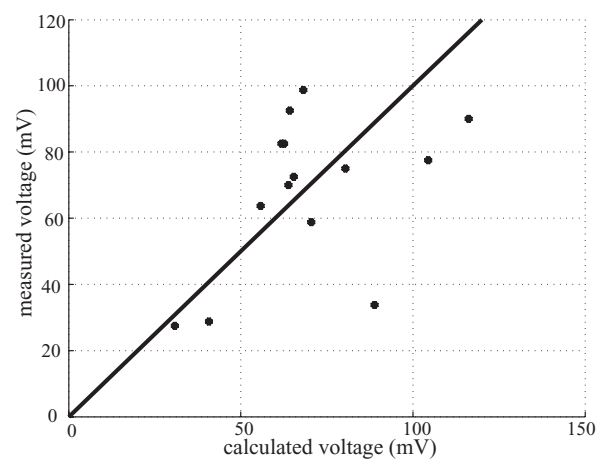

(c)

Fig. 8. Comparison of the calculated and measured voltage values for the activation of: (a) $z$-gradient coil, (b) $y$-gradient coil, and (c) $x$-gradient coil. (Note that implant leads are aligned in the $x$-axis along the body). $x$-direction, the root-mean-square error is calculated as $25 \mathrm{mV}$. In order to verify that the provided expressions are independent from the conductivity, experiments are repeated for different conductivity values and measurements at the same lead positions are noted. For these experiments, conductivity values are measured as $0.074,0.25,0.35$, and $0.44 \mathrm{~S} / \mathrm{m}$. For five different lead positions, similar measurement results are obtained with a $3-\mathrm{mV}$ root mean square error. Note that in this study, the analytical calculations and the experiments are done for only a homogeneous body model and no comparison is given between homogeneous and heterogeneous body models unlike the experiments in [5]. However, if the field calculations cannot be done for each patient specifically, there will always be calculation errors; therefore, simplified expressions may suffice to obtain approximate values to assess the stimulation risk.

In ex vivo experiments, six frogs are used and the stimulation risk is observed at 40 different lead positions. The threshold voltage value for stimulating the frog nerve is measured as $0.1 \mathrm{~V}$ outside of the MR scanner. During MR experiments, coordinates of each lead position is determined by MR images, and for each case the approximate induced voltage values are calculated for $x-, y$-, and $z$-gradient coils. It is seen that for 24 lead positions the calculated voltage values are between 0.11 and $0.3 \mathrm{~V}$. These values are bigger than the measured threshold voltage and stimulation is observed at these lead positions as expected. For the remaining 16 lead positions, the calculated voltage values are between 0.01 and $0.098 \mathrm{~V}$. These values are smaller than the measured threshold voltage; hence, stimulation is not expected. However, in the two lead positions where the calculated voltages are 0.094 and $0.098 \mathrm{~V}$, stimulation is also observed. Therefore, we decided to set an approximate threshold voltage level as $0.09 \mathrm{~V}$ allowing a $10 \%$ difference with the measured one. Note that this $10 \%$ difference can be attributed to 5-10 $\mathrm{mV}$ error margin as mentioned before. A similar difference is also observed in phantom experiments where the measured voltages are slightly higher than the calculated ones for some lead locations.

The error in these simplified expressions needs to be investigated for noncylindrical and heterogeneous objects like human body. Furthermore, in this study, we assumed that the implantable pulse generator (IPG) case is directly connected to the 
lead. Although this may be considered as a worst case condition, the impedance between the lead and the IPG and the other circuit elements (e.g., EMI capacitors) used to enhance the MRI compatibility of the AIMD can also be put into the model and with this model gradient induced current passing through the lead can be calculated with the knowledge of the induced voltage. This analysis with experimental verifications is planned as a future study.

\section{CONCLUSION}

In this study, we derived simplified expressions for the electric field inside the cylindrical homogeneous body model for a perfectly uniform gradient field. These simple expressions may be used to understand the nerve stimulation risk when there is an implant. Both phantom and ex vivo experiments are performed and results show that if the path of the implant lead is known, the induced voltage on the lead can be estimated analytically.

\section{REFERENCES}

[1] J. P. Reilly, "Peripheral nerve stimulation by induced electric currents: Exposure to time-varying magnetic fields," Med. Biol. Eng. Comput., vol. 27, no. 2, pp. 101-110, 1989.

[2] D. Buechler, C. Durney, and D. Christensen, "Calculation of electric fields induced near metal implants by magnetic resonance imaging switched gradient magnetic fields," Magn. Res. Imag., vol. 15, no. 10, pp. 11571166, 1997.

[3] H. Tandri, M. M. Zviman, T. Lloyd, S. R. Wedan, R. D. Berger, and H. Halperin, "Determinants of gradient field-induced current in a pacemaker lead system in a magnetic resonance imaging environment," Heart Rhythm, vol. 5, no. 3, pp. 462-468, 2008.

[4] H. I. Bassen and G. G. Mendoza, "In-vitro mapping of e-fields induced near pacemaker leads by simulated MR gradient fields," BioMedical Eng. OnLine, vol. 8, no. 1, pp. 1-18, 2009.

[5] F. Liu, H. Zhao, and S. Crozier, "On the induced electric field gradients in the human body for magnetic stimulation by gradient coils in MRI," IEEE Trans. Biomed. Eng., vol. 50, no. 7, pp. 804-815, Jul. 2003.

[6] P. P. M. So, M. A. Stuchly, and J. A. Nyenhuis, "Peripheral nerve stimulation by gradient switching fields in magnetic resonance imaging," IEEE Trans. Biomed. Eng., vol. 51, no. 11, pp. 1907-1914, Nov. 2004.
[7] J. A. D. Boer, J. D. Bourland, J. A. Nyenhuis, C. L. G. Ham, J. M. L. Engels, F. X. Hebrank, G. Frese, and D. J. Schaefer, "Comparison of the threshold for peripheral nerve stimulation during gradient switching in whole body MR systems," IEEE Trans. Biomed. Eng., vol. 15, no. 5, pp. 520-525, 2002.

[8] H. Zhao, S. Crozier, and F. Liu, "Finite difference time domain (FDTD) method for modeling the effect of switched gradients on the human body in MRI," Magn. Reson. Med., vol. 48, pp. 1037-1042, 2002.

[9] A. Hoffmann, S. Faber, K. Werhahn, L. Jager, and M. Reiser, "Electromyography in MRI first recordings of peripheral nerve activation caused by fast magnetic field gradients," Magn. Reson. Med., vol. 43, pp. 534-539, 2000.

[10] B. A. Chronik and M. Ramachandran, "Simple anatomical measurements do not correlate significantly to individual peripheral nerve stimulation thresholds as measured in MRI gradient coils," Magn. Reson. Med., vol. 17, pp. 716-321, 2003.

[11] J. P. Reilly, "Magnetic field excitation of peripheral nerves and the heart: A comparison of thresholds," Med. Biol. Eng. Comput., vol. 29, pp. 571$579,1991$.

[12] P. M. Glover and R. Bowtell, "Measurement of electric fields induced in a human subject due to natural movements in static magnetic fields or exposure to alternating magnetic field gradients," Phys. Med. Biol., vol. 53, no. 2, pp. 361-373, 2008.

[13] R. Bowtell and R. Bowley, "Analytic calculations of the e-fields induced by time varying magnetic fields generated by cylindrical gradient coils," Magn. Reson. Med., vol. 44, pp. 782-790, 2000.

[14] F. Liu, L. Xia, and S. Crozier, "Influence of magnetically-induced e-fields on cardiac electric activity during MRI: A modeling study," Magn. Reson. Med., vol. 50, no. 6, pp. 1180-1188, 2003.

[15] J. P. Reilly and A. M. Diamant, "Theoretical evaluation of periphera nerve stimulation during MRI with an implanted spinal fusion stimulator," Magn. Res. Imag., vol. 15, no. 10, pp. 1145-1156, 1997.

[16] F. Rattay, "Analysis of models for the external stimulation of axons," IEEE Trans. Biomed. Eng., vol. 33, no. 10, pp. 974-977, Oct. 1986.

[17] J. Jin, Electromagnetic Analysis and Design in Magnetic Resonance Imaging. Boca Raton, FL: CRC Press, 1999

[18] R. Turner, "Gradient coil design: A review of methods," Magn. Res. Imag., vol. 11, pp. 903-920, 1993.

[19] M. Abramowitz and I. A. Stegun, Handbook of Mathematical Functions. New York: Dover, 1965.

[20] E. Abaci, E. Kopanoglu, V. B. Erturk, and E. Atalar, "Simple analytical equation of the induced e-field," presented at the Int. Society for Magnetic Resonance in Medicine, Toronto, Canada, 2008.

Author, photographs and biographies not available at the time of publication. 